\title{
Killer Knots: Molecular evolution of Inhibitor Cystine Knot toxins in wandering spiders (Araneae: Ctenidae)
}

\author{
T. Jeffrey Cole, Michael S. Brewer
}

\begin{abstract}
Venom expressed by the nearly 50,000 species of spiders on Earth largely remains an untapped reservoir of a diverse array of biomolecules with potential for pharmacological and agricultural applications. A large fraction of the noxious components of spider venoms are a functionally diverse family of structurally related polypeptides with an inhibitor cystine knot (ICK) motif. The cysteine-rich nature of these toxins makes structural elucidation difficult, and most studies have focused on venom components from the small handful of medically relevant spider species such as the highly aggressive Brazilian wandering spider Phoneutria nigriventer. To alleviate difficulties associated with the study of ICK toxins in spiders, we devised a comprehensive approach to explore the evolutionary patterns that have shaped ICK functional diversification using venom gland transcriptomes and proteomes from phylogenetically distinct lineages of wandering spiders and their close relatives. We identified 626 unique ICK toxins belonging to seven topological elaborations. Phylogenetic tests of episodic diversification revealed distinct regions between cysteine residues that demonstrated differential evidence of positive or negative selection, which may have structural implications towards the specificity and efficacy of these toxins. Increased taxon sampling and whole genome sequencing will provide invaluable insights to further understand the evolutionary processes that have given rise to this diverse class of toxins.
\end{abstract}

\section{Introduction}

Animals of numerous phyla have independently evolved venom to inject into and cause harm to other animals for the purposes of either subduing prey, fending off predators, or competing with rival mates ${ }^{1-3}$. Venomous animals constitute $\sim 15 \%$ of all described animal biodiversity, with spiders representing the largest group of venomous animals with approximately $\sim 50,000$ species currently described ${ }^{4,5}$. Recent phylogenetic investigations have revealed that spiders recruited inhibitor cystine knot (ICK) toxins into their venom arsenal via a duplication event and subsequent neofunctionalization $\sim 300$ million years ago ${ }^{6}$. Several subsequent rounds of duplication events and subfunctionalization throughout their evolutionary history has given rise to a vast library of toxins that has allowed spiders to succeed as generalist predators that can incapacitate a broad array of prey items by expressing hundreds to thousands of unique venom components spanning an estimated million unique pharmacologically active components spread across all species of spiders ${ }^{7-9}$.

The exploration of venom components in spiders has largely focused on the small handful of spiders with dangerous bites to humans, which has greatly limited and biased the current understanding and biological context of the diverse array of toxins expressed by spiders. A prime example is the medically relevant Brazilian wandering spider Phoneutria nigriventer, which has been the focus of numerous investigations to delineate active noxious components ${ }^{10,11}$. One such component is toxin Tx2-6 which is the responsible agent for priapism, occasionally an envenomation symptom of Phoneutria in human males ${ }^{12}$. The most notable component of their venom is toxin Tx1, which exerts inhibitory effects on neuronal sodium channels in a highly selective manner and has a lethal median dosage $\left(\mathrm{LD}_{50}\right)$ of $47 \mu \mathrm{g} / \mathrm{kg}$ in Mus musculus ${ }^{13}$. This is nearly four times more toxic than the lethal nerve agent Sarin, which is classified as a Schedule 1 substance by the Chemical Weapons Convention of 1993 and has an $\mathrm{LD}_{50}$ of $172 \mu \mathrm{g} / \mathrm{kg}$ in M. musculus when injected 
subcutaneously ${ }^{14,15}$.

A recent investigation of the venom gland transcriptome and proteome of $P$. nigriventer (Keyserling, 1891) revealed that their noxious venom components belong to a diverse class of ICK toxins ${ }^{16}$. The core ICK cysteine framework consists of three pairs of cysteines forming disulfide bridges between $C_{1}-C_{4}, C_{2}-C_{5}, C_{3}-C_{6}$ to take on an unusually stable conformation. That same investigation recovered 98 cysteine rich toxins that represented nine additional cysteine frameworks, six of which were verified to be ICKs. The number of cysteine residues per group ranged from six to fourteen. They also spanned a broad range of predicted functionality, from ion channel modulators of varying specificity $\left(\mathrm{Ca}^{+2}, \mathrm{~K}^{+}\right.$, and $\left.\mathrm{Na}^{+}\right)$, to protease inhibitors and NMDA receptor modulators. The cysteine-rich peptide toxins represented $93.24 \%$ of the relative abundance of peptides expressed in the venom when accounting for expression levels.

The discovery of a vast library of ICK toxins expressed by $P$. nigriventer has led to numerous questions about their evolutionary origins. In this study, we used venom gland transcriptomics and proteomics with increased taxon sampling from phylogenetically distinct lineages to provide the first comprehensive framework to test hypotheses about the molecular evolution of ICK toxins in wandering spiders.

\section{Methods}

Taxon sampling: Three adult males and females were collected from all members of Ctenidae in the United States, excluding the narrow Texas cave endemic C. valveriensis. Specimens of C. hibernalis, C. exlineae, $C$. captiosus, L. byrrhus, and A. punctula were collected from the following respective localities: North-Central Alabama (33.462, -86.788), Northwest Arkansas (34.376, -94.029), Central Florida (29.0823, -81.578), SouthCentral Texas (29.831, -99.573), and Northwest Georgia (34.049, -85.381). Four male and female Phoneutria nigriventer along with four female Isoctenus sp. supplied by a Brazilian collaborator. Ctenidae belongs to the superfamily Lycosoidea, which is a member of a clade of spiders that possess a retrolateral tibial apophysis (RTA), a backward-facing projection on the tibia of the male pedipalp. To allow for an investigation of the broader evolutionary context of ICK toxins in wandering spiders, whole body transcriptomes from outgroups within the RTA clade were retrieved from NCBI's Short Read Archive.

Venom and RNA isolation: Spiders were housed in a $500 \mathrm{~cm}^{3}$ plastic container and were watered, cleaned and fed crickets (Acheta domesticus) weekly. The temperature was maintained between $72-77^{\circ} \mathrm{C}$, which is near the average temperature that these animals experience in their natural habitats. Prior to venom collection, individuals were anesthetized with $\mathrm{CO}_{2}$ using a modified procedure as described by Barrio \& Brazil [17]. Venom was collected using electrostimulation with $7 \mathrm{~V}$ of AC current, similar to previous studies ${ }^{18-20}$. Anesthetized individuals were placed on clamped forceps attached to an electrode. One prong of the forceps was wrapped in non-conductive insulating tape to create a point of contact for the spider that retards current, while the other prong of the forceps was wrapped with a cotton thread and soaked in saline to create a point of contact with the spider to promote electrical conductivity. A capillary tube was then placed over the fang in order to collect the venom. Finally, the second electrode was placed on a syringe connected to a vacuum pump which was touched to the base of the chelicerae in order to complete the circuit and allow the muscles around the venom gland to contract and eject venom into the capillary tube while simultaneously allowing regurgitate to be vacuum pumped through the syringe to prevent contamination. The collected venom was then stored at $-80^{\circ} \mathrm{C}$. Two days after venom milking, the venom glands of each ctenid were dissected out, whole RNA was isolated from the venom glands from the five U.S species and the two Brazilian species using TRIzol® (Life Technologies, Carlsbad, CA) and the Qiagen RNeasy kit (Qiagen, Valencia, CA). RNA concentration and integrity was evaluated using Quant-iT ${ }^{\mathrm{TM}}$ PicoGreen and Bioanalyzer.

Sequencing and processing: The RNA extractions were sent to the Genomic Services Lab at HudsonAlpha (Huntsville, AL) for library preparation with poly(A) selection and sequencing on a 100 bp paired-end run on an Illumina HiSeq 2500, comprising 25 million reads forward and reverse (50 million total reads) per sample. For all samples sequenced after 2018, due to sequence facility updates, the same setup was used but with Illumina NovaSeq. Additionally, RNAseq reads from all outgroup species were retrieved from NCBI Short Read Archive.

Prior to assembly, FASTP v $0.19 .6^{21}$ was used to remove adapters, correct sequencing errors, and trim 
low-quality base calls, ensuring maximal accuracy of transcript recovery ${ }^{22}$. All read processing was executed with. De novo assemblies typically recover an unexpectedly large number of transcripts, sometimes well over $100,000^{23}$. This happens for three main reasons. First, an increased depth of sequencing combined with improved transcript recovery algorithms increases the recovery of transcripts that are expressed at levels lower than would otherwise be considered biologically relevant. Second, common contaminants, such as bacteria and fungi, unavoidably make their way into samples, thus inflating the mRNA transcript pool diversity. Third, in eukaryotic systems, alternative splicing yields a significant increase in recoverable transcripts per gene locus as isoforms. Further, when several RNA-seq experiments from different species are sequenced together, cross contamination inevitably occurs ${ }^{24-30}$. The aforementioned issues can have tremendous effects on downstream phylogenetic inferences made from problematic transcripts ${ }^{31}$. To alleviate these issues, reads were first mapped to a transcript database of common contaminants (bacteria, fungi, human, and nematodes) using salmon v1.3.0 with default mapping parameters, all unmapped reads were retained as the finalized library of processed reads ${ }^{32}$.

Transcript reconstruction and expression quantification: The processed reads were de novo assembled into transcripts using TRINITY v2.8.4 $4^{33}$. An important aspect of elucidating the functional relevance of a given protein is the quantification of its expression level. This can be achieved at the transcript level through quantifying read coverage by mapping the reads from each sample back to their assembled transcripts. Salmon uses a quasi-mapping approach and is one of the fastest, most efficient, and most accurate methods for quantifying expression in RNAseq experiments ${ }^{32}$. Since TRINITY also assembles splice variants and alleles of the same gene, these were consolidated into SuperTranscripts ${ }^{34}$ prior to mapping so that the inferred expression values are at the gene level and not the isoform level. SuperTranscripts are formed by collapsing common and unique regions of sequences among splicing isoforms into a singular consolidated linear sequence. Then, the SuperTranscripts were used as mapping targets, and the processed reads were pseudomapped using salmon to quantify expression levels as Transcripts per million (TPM).

Venom proteomics: To characterize the venom profiles, venom proteins were isolated using HPLC followed by MALDI-TOF Mass Spectrometry. MALDI-TOF is the preferred method for mass analysis in proteins due to only singly charging analytes, compared to commonly used ESI techniques which apply multiple charges to analytes and complicate downstream analysis. The finalized dataset of candidate venom encoding transcripts were elucidated by cross-referencing proteomic MS data to the transcriptome using the CRUX pipeline ${ }^{35}$.

Locus sampling: Coding sequences within transcripts were inferred using TRANSDECODER v3.0.1 $1^{36}$. TRANSDECODER uses the following criteria to identify the single best coding sequence in a given transcript: available open reading frame (ORF) of a minimum length of 30 codons, log-likelihood score of the coding sequence, predictions of start and stop codons as refined by a Position-Specific Scoring Matrix. The completeness of the assemblies was evaluated using BUSCO v3.0.2 (Benchmarking Universal Single-Copy Orthologs) $)^{37}$ with the arthropod database. In addition to providing a metric of assembly completeness, complete BUSCO transcripts serve as a robust set of loci for phylogenetic analysis.

Phylogenetic reconstruction: A well supported phylogeny provides a necessary evolutionary framework for comparative analysis of venom evolution. To reconstruct the North American ctenid species relationships, additional RNAseq reads from 20 outgroup species were retrieved from NCBI Short Read Archive. Loci sampling for phylogenetic analysis involved the following procedure. Only complete coding sequences inferred from TRANSDECODER that were the longest isoform of a given TRINITY gene assignment were used for this analysis. Coding sequences that contained a single complete match to a BUSCO term were retrieved from the assemblies. Multiple protein alignments were generated with MAFFT v7.221 ${ }^{38}$ for BUSCO matches and retained if 30 out of 48 samples were present and $<50 \%$ of the sequences were identical. This resulted in 245 BUSCO term alignments that were then trimmed with TRIMAL v1.4. ${ }^{39}$ to remove uninformative sites. Model selection was performed for the trimmed concatenated matrix to elucidate the best-fit model using the "TESTONLY" option of IQ-TREE v1.6.1040. Subsequently, 1,000 ultrafast bootstrap replicates were performed to calculate node support. Additionally, site and gene concordance factors were calculated as alternative support metrics. For the species tree analysis, the phylogenies of each gene were reconstructed using IQTREE with the same settings as previously mentioned ${ }^{40}$. The gene trees were then provided as input for ASTRAL v2.0 $0^{41}$ to reconstruct the species phylogeny. 
Inhibitor cystine knot annotation: To identify inhibitor cystine knot toxins in the transcriptome assemblies, a database of verified ICKs from spiders was retrieved from the KNOTTIN database ${ }^{42}$. Only ICKs with complete coding sequences and verified disulfide connectivity were retained in the final verified ICK database. The database was provided as input to BLASTp to search against the inferred protein sequences from TRANSDECODER ${ }^{43}$. Additionally, a multiple sequence alignment was generated from the verified ICK database to create a Hidden Markov Model (HMM) that could be searched against the genomic protein sequences using HMMER v3.3.144. For both BLASTp and HMMER, only matches with at least six cysteines and up to 200 amino acids in length were kept for downstream analysis. Additionally, putative matches were only kept if they contained a signal peptide as indicated by signalP v5.0, which predicts the presence of signal peptide cleavage sites ${ }^{45}$. A homology network of the finalized peptides was generated using an all against all BLASTp search, and then provided as input to SiLiX v1.2.11 to group the peptides into putative gene families ${ }^{46}$. Cysteine frameworks were designated using the following approach. Cysteines that were directly adjacent to each other were designated as $C C$. Cysteines separated by one residue were denoted as $C X C$. Finally, cysteines separated by more than one residue were designated as $C-C$. Each framework was given a numeric code to represent the number of cysteines they contain along with a unique identifier. To ensure that only ICKs are included in the analysis, only cysteine frameworks representing the top $80 \%$ of peptides in the largest family were included for downstream disulfide connectivity predictions and phylogenetic analysis. A non-redundant dataset was then created to only include unique coding sequences.

Disulfide connectivity predictions: Disulfide connectivity is of great importance in understanding structural homology in ICK toxins when sequence similarity is greatly reduced. Determining disulfide connectivity normally requires empirical structural validation but can be reasonably predicted using computational predictions. The number of possible disulfide bonds explodes in a combinatorial fashion, to the point where exhaustively comparing disulfide connection possibilities in peptides with more than 6 cysteines is not computationally tractable ${ }^{47}$. To alleviate this, a number of heuristic approaches have been developed. For this dataset the following four approaches were used to generate disulfide connection predictions for a random representative mature peptide from the top cysteine frameworks.

1. DISULFIND collectively decides the bonding state assignment of the entire chain using a Support Vector Machine binary classifier followed by a refinement stage ${ }^{48}$. DISULFIND v1.1 was used to generate a total of three alternative disulfide connection predictions.

2. CYSCON uses a hierarchical order reduction protocol to identify the most confident disulfide bonds and then evaluate what remains using Support Vector Regression ${ }^{49}$. CYSCON v2015.09.27 was used to generate a single disulfide prediction per ICK representative per unique cysteine framework.

3. CRISP v1.0 not only predicts disulfide bonds, but also the entire structure of a cysteine rich peptide by searching a customized template database with cysteine-specific sequence alignment with three separate machine learning models to filter templates, rank models, and estimate model quality ${ }^{50}$. CRISP was used to generate five structural models for each ICK representative per unique cysteine framework.

A chord diagram was constructed for each cysteine framework to demonstrate the variability in disulfide connectivities for every prediction attempt of each of the approaches using the D3 JavaScript library ${ }^{51}$. A consensus disulfide connection prediction of all the approaches in conjunction with previously published disulfide connections, as found through Arachnoserver ${ }^{52}$, were used to generate the finalized disulfide connectivity predictions for the three disulfide bridges homologous to all cysteine frameworks.

Phylogenetic tests for selection: Aligning ICKs, or any cysteine rich peptide, is difficult due to nonhomologous cysteines mistakenly being aligned. Thus, the finalized consensus disulfide connectivities were used to inform the alignment of ICKs using a similar approach to Pineda et al. [6]. Rather than align everything at once and then manually adjusting misaligned cysteines followed by realignment of regions between the two adjusted cysteines, only amino acids between cysteines participating in disulfide bonds common to all ICKs in the dataset were used for the alignment. Additionally, the regions between homologous cysteines were aligned separately while using the barcode "WWYHWYYHMM" to replace flanking cysteines to prevent inner cysteines from misaligning with flanking cysteines similar to the approach by ${ }^{53}$. The alignment was then provided as input to IQTREE v1.5.5 $5^{40}$ to test the amino acid composition using a Chi-squared test. Any sequences that failed the test were removed from the alignment, and the sub-regions were realigned following 
the previously described procedure. The resulting alignment was reverse translated to form a coding sequence alignment using PRANK ${ }^{54}$.

The same outgroup as used by Pineda et al. [6] (disulfide-directed $\beta$-hairpin from the whip scorpion Mastigoproctus giganteus) was added to the protein alignment using MAFFT v7.455 ${ }^{38}$. The phylogenetic relationships of the ICKs in this alignment were reconstructed using IQTREE and the default settings.

Adaptive molecular evolution is typically inferred in coding sequences by comparing ratios of the rates of nonsynonymous substitution and synonymous substitution $\left(d_{N} / d_{S}\right.$ or $\left.\omega\right)$, where $d_{N}$ exceeding $d_{S}$ indicates positive selection, $d_{S}$ exceeding $d_{N}$ indicates negative selection, and $d_{N} / d_{S}$ approaching unity indicates neutral evolution. One of the broadest questions to ask in regards to how a gene has evolved is "Has a particular gene evolved under positive selection?" To do this, the HYPHY ${ }^{55}$ implementation of Branch-Site Unrestricted Statistical Test (BUSTED) for Episodic Diversification assesses whether a gene has experienced positive selection at at least one site on at least one branch, by fitting a codon model with three rate classes constrained as $\omega_{1} \leq \omega_{2} \leq 1 \leq \omega_{3}{ }^{56}$. This unconstrained model is compared to a null model disallowing positive selection where $\omega_{3}=1$. To determine if ICKs have experienced positive selection, the codon multiple sequence alignment and phylogeny were provided as input to BUSTED using default parameters with the entire phylogeny set as the background.

In ICKs, specific amino acid sites may play an important role in the structure-function (e.g., binding specificity) and adaptive evolution. To identify specific amino acid sites that have undergone pervasive positive selection, the HYPHY implementation of a Fast, Unconstrained Bayesian AppRoximation (FUBAR) employs a pre-specified discrete grid of $d_{N}$ and $d_{S}$ values to be applied across sites ${ }^{57}$. The codon multiple sequence alignment and phylogeny were provided as input to FUBAR with default parameters.

There may only be specific episodes where certain amino acids receive strong bouts of positive selection. To determine if a certain number of branches have amino acid sites undergoing positive selection, the HYPHY implementation of a Mixed Effects Model of Evolution (MEME) ${ }^{58}$ by comparing a null model of rate parameters to an alternative model of rate parameters at each branch. Both models include a single $d_{S}$ or $\alpha$ value along with two separate $d_{N}$ or $\beta$ values $\left(\beta^{+}\right.$and $\beta^{-}$), where $\alpha$ is shared between both $\beta$ values per site. In the null model, both $\beta$ values are restricted to be less than or equal to $\alpha$, whereas $\beta^{+}$is unrestricted in the alternative model. Positive selection is inferred when $\beta^{+}>\alpha$. To determine if certain amino acid sites have undergone episodic positive selection, the codon multiple sequence alignment and phylogeny were provided as input to MEME with default parameters and the entire phylogeny set as the background.

To evaluate specific instances on a phylogeny where positive selection has occurred, branch-site models are typically implemented. Much like how MEME is unable to statistically specify the exact branches within a site undergoing episodic positive selection, branch-site models are only able to identify specific branches where a certain portion of sites have undergone positive selection. This can be accomplished using the HYPHY implementation of adaptive Branch-Site Random Effects Likelihood (aBSREL) ${ }^{59}$, by modeling both site-level and branch-level $\omega$ heterogeneity. Since some branches may feature more or less complex evolutionary patterns, aBSREL infers the optimal number of $\omega$ rate classes for each branch using the sample Akaike information criterion $\left(A I C_{c}\right)$. A likelihood ratio test is then performed to compare the full adaptive model to a null model where branches are not allowed to have rate classes where $\omega$ exceeds unity.

Aside from evaluating signatures of positive selection through calculations of codon substitution rates, we also investigated the co-occurrence between amino acid positions in ICKs, which may provide useful inferences into the evolution of their structure/function. This can be achieved using the HYPHY implementation of the Bayesian Graphical Model (BGM) ${ }^{60}$, which maps amino acid substitutions to a phylogeny and reconstructs ancestral states for a given model of codon substitution rates that is then followed up by a series of $2 \times 2$ contingency table analyses.

\section{Results}

Venom gland transcriptome and proteome: The 48 assemblies in this analysis included an average transcript recovery of $106,410(\mathrm{~s} . \mathrm{d}=43,708)$, representing an average of $84,474(\mathrm{~s} . \mathrm{d}=32,908)$ genes as designated by 
Trinity (Table 1). The 21,936 genes with alternative transcripts designated by Trinity had an average of 3.04 isoforms $(\mathrm{s.d}=1.99)$. From the longest isoforms, we recovered on average 12,173 complete coding sequences per species $(\mathrm{s} . \mathrm{d}=4,993)$, with an average amino acid length of $264(\mathrm{~s} . \mathrm{d}=9)$.

Table 1: Transcriptome assembly statistics for all ctenid samples contributed to this study, including number of SuperTranscripts and coding sequences.

\begin{tabular}{llllll}
\hline species & sex & sample & transcripts & superTranscripts & CDS \\
\hline Anahita punctulata & male & 297 & 120,048 & 88,950 & 55,238 \\
Ctenus captiosus & female & 305 & 124,919 & 99,712 & 59,434 \\
Ctenus captiosus & female & 311 & 140,647 & 110,550 & 64,437 \\
Ctenus captiosus & male & 303 & 157,109 & 123,061 & 70,951 \\
Ctenus captiosus & male & 306 & 158,795 & 122,878 & 71,795 \\
Ctenus exlineae & female & 244 & 105,140 & 85,561 & 49,630 \\
Ctenus exlineae & female & 245 & 111,981 & 89,516 & 52,365 \\
Ctenus exlineae & female & 247 & 112,224 & 90,112 & 52,434 \\
Ctenus exlineae & male & 242 & 95,088 & 78,974 & 44,942 \\
Ctenus exlineae & male & 246 & 54,776 & 46,375 & 24,166 \\
Ctenus hibernalis & female & 91 & 194,576 & 148,947 & 83,512 \\
Ctenus hibernalis & female & 92 & 161,519 & 124,108 & 71,340 \\
Ctenus hibernalis & male & 148 & 202,764 & 157,422 & 81,381 \\
Leptoctenus byrrhus & female & 136 & 99,257 & 81,488 & 47,189 \\
Leptoctenus byrrhus & male & 213 & 108,687 & 88,723 & 50,313 \\
Leptoctenus byrrhus & male & 222 & 101,706 & 83,634 & 47,527 \\
\hline
\end{tabular}

Phylogenetic results: The average percentage of complete BUSCO hits within the assemblies was $84.96 \%$ (s.d $=15.48 \%)$, and an average of $60.33 \%$ ( $. d=8.90 \%)$ were single copy. 245 BUSCO loci met the threshold of $60 \%$ of species represented with at least $50 \%$ of the sequences being nonidentical. The untrimmed alignments had an average matrix width of 348.8 nucleotides $(\mathrm{s} . \mathrm{d}=179.0)$; trimming the alignments reduced the average to 314.7 nucleotides $(\mathrm{s} . \mathrm{d}=162.8)$. The total size of the concatenated matrix was 78,594 nucleotides.

There were no topological differences between the concatenated matrix phylogeny and the ASTRAL species tree. The only topological difference that occurred between this study and Cheng \& Piel [61] was that we recovered Oxyopidae as sister to a clade comprising Ctenidae + Psechridae + Lycosidae + Pisauridae, instead of being sister to the Thomisidae. Temperate zone North American ctenids do not form a single lineage, as Anahita punctulata is sister to a clade comprising the North American Ctenus-Leptoctenus lineage plus the Neotropical Phoneutria-Isoctenus clade. Within the Ctenidae, the genus Ctenus is polyphyletic with respect to Ctenus corniger, the only Old World representative of the family, being sister to all New World member ctenids included here (Figure 1). This recapitulates previous findings that Ctenus has served as a repository for taxonomically problematic species ${ }^{62-65}$.

Inhibitor cystine knot annotation: A total of 1,259 cysteine rich peptides were recovered that met the following criteria: signal peptide present, less than 200 amino acids, mature peptide had at least 6 cysteines, and was a match with the KNOTTIN database from either a BLAST search or HMMER with an e-value cutoff of $1 \times 10^{-3}$. On average each sample contained 26.6 cysteine rich peptides $(\mathrm{s} . \mathrm{d}=8.3)$. Cysteine rich peptides made up less than $10 \%$ of the total peptides in the proteomes of C. exlineae and C. hibernalis, though in at least two samples they made up over $50 \%$ of the relative abundance when accounting for expression level (Table 2). 
bioRxiv preprint doi: https://doi.org/10.1101/2021.07.28.453581; this version posted July 28, 2021. The copyright holder for this preprint (which was not certified by peer review) is the author/funder, who has granted bioRxiv a license to display the preprint in perpetuity. It is made available under aCC-BY-NC-ND 4.0 International license.

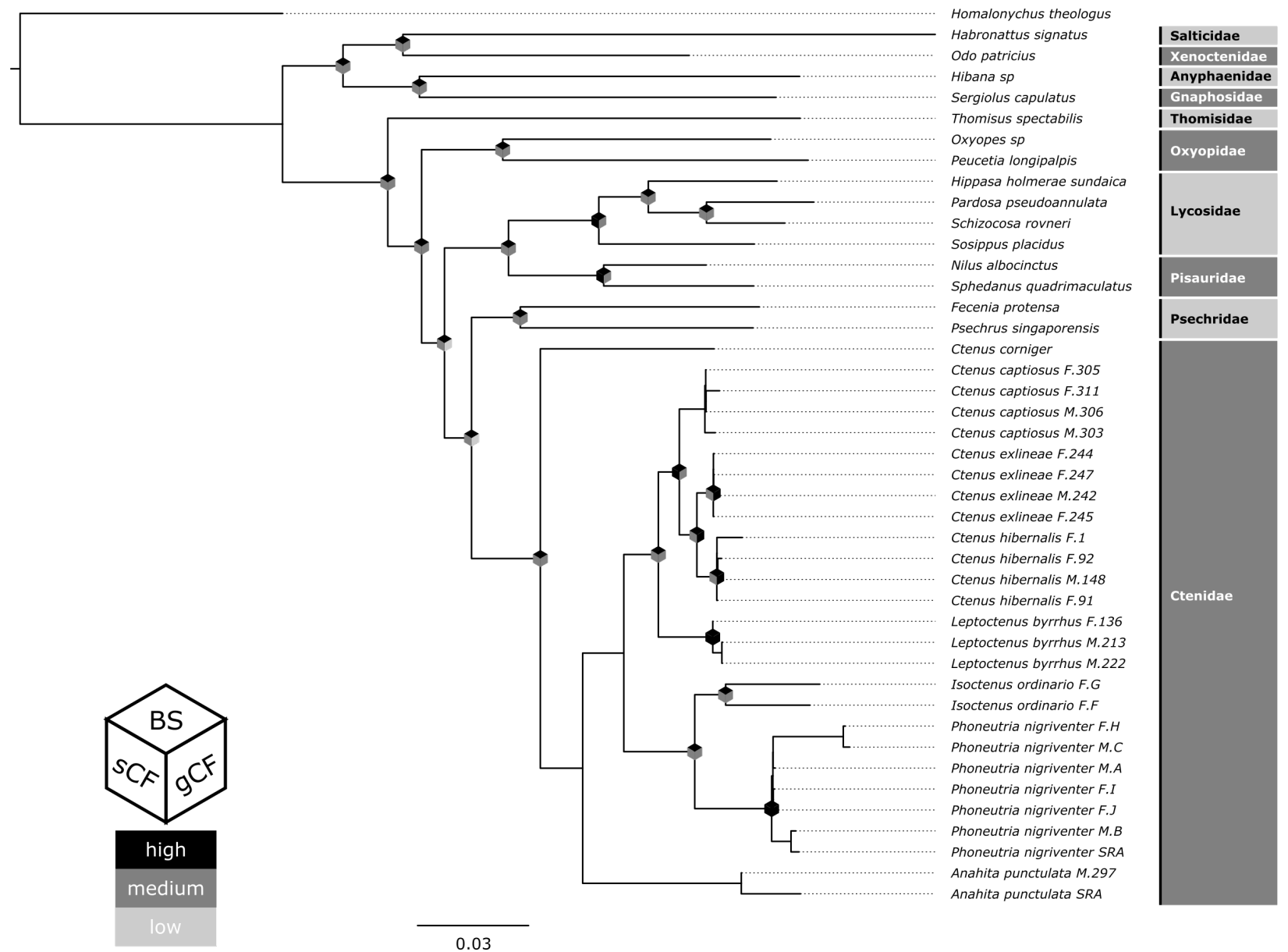

Figure 1: Reconstructed species-level phylogeny from concatenated matrix using IQTREE. Bootstrap support, as well as gene and site concordance factor values are indicated by the color of the diamond placed on the inner nodes. Black, dark grey and grey indicate high, medium, and low support, respectively. Cutoff values for bootstrap: $70-100 \%, 50-70 \%, 0-50 \%$. Cutoff values for gene concordance factors: $70-100 \%, 20-70 \%, 0-20 \%$. Cutoff values for site concordance factors: $60-100 \%, 33-60 \%, 0-33 \%$. 
Table 2: Cysteine rich peptide composition in the proteomes of $C$. exlineae and $C$. hibernalis, with comparison to the expression levels from the venom gland transcriptomes of each sample per species.

\begin{tabular}{lllllllll}
\hline species & sex & sample & peptides & ICKs & \%ICK & sum TPM & ICK TPM & \%TPM \\
\hline C. exlineae & male & 242 & 113 & 5 & $4.42 \%$ & $18,214.3$ & $9,670.5$ & $53.1 \%$ \\
C. exlineae & female & 245 & 127 & 7 & $5.51 \%$ & $16,755.8$ & $7,002.0$ & $41.8 \%$ \\
C. exlineae & male & 246 & 200 & 13 & $6.50 \%$ & 131,454 & $51,011.1$ & $38.8 \%$ \\
C. exlineae & female & 244 & 339 & 5 & $1.47 \%$ & $42,546.4$ & $12,227.6$ & $28.7 \%$ \\
C. exlineae & female & 247 & 194 & 5 & $2.58 \%$ & $27,378.7$ & $5,436.3$ & $19.9 \%$ \\
C. hibernalis & female & 4926 & 196 & 8 & $4.08 \%$ & 109,103 & $57,283.2$ & $52.5 \%$ \\
C. hibernalis & female & 91 & 525 & 12 & $2.29 \%$ & 111,759 & $14,703.9$ & $13.2 \%$ \\
C. hibernalis & male & 148 & 170 & 7 & $4.12 \%$ & $31,121.2$ & $2,581.5$ & $8.3 \%$ \\
C. hibernalis & female & 92 & 176 & 5 & $2.84 \%$ & $37,128.6$ & $2,366.6$ & $6.4 \%$ \\
\hline
\end{tabular}

SiLiX grouped the cysteine rich peptides into 53 putative gene families. The largest family comprised 1,148 peptides, and the top seven frameworks corresponding to the ICKs described by Diniz et al. [16] represented 960 putative ICKs. The largest cysteine framework recovered was C8.0 with 538 peptides, whereas C6.0, the next largest, represented 123 peptides (Table 3).

Table 3: Summary of the number of peptides recovered per cysteine framework as well as the corresponding numeral indication designated by Diniz et al. [16].

\begin{tabular}{llll}
\hline & Dinez & & \\
identifier & Numeral & motif & total \\
\hline 6.0 & I & $C_{1}-C_{2}-C_{3} C_{4}-C_{5}-C 6$ & 123 \\
8.0 & II & $C_{1}-C_{2}-C_{3} C_{4}-C_{5} X C 6-C_{7} X C_{8}$ & 538 \\
10.0 & $\mathrm{~V}$ & $C_{1}-C_{2}-C_{3} X C_{4} C_{5}-C 6 X C_{7}-C_{8} X C_{9}-C 10$ & 117 \\
10.1 & & $C_{1}-C_{2}-C_{3} C_{4} C_{5}-C 6 X C_{7}-C_{8} X C_{9}-C 10$ & 23 \\
12.0 & VI & $C_{1}-C_{2}-C_{3} X C_{4} C_{5}-C 6 X C_{7}-C_{8} X C_{9}-C_{10}-C_{11}-C_{12}$ & 100 \\
12.1 & VII & $C_{1}-C_{2}-C_{3} X C_{4} C_{5} X C 6-C_{7} X C_{8}-C_{9} X C_{10}-C_{11}-C_{12}$ & 27 \\
14.0 & VIII & $C_{1}-C_{2}-C_{3} X C_{4} C_{5}-C 6 X C_{7}-C_{8} X C_{9}-C_{10}-C_{11}-C_{12}-C_{13}-C_{14}$ & 33 \\
\hline
\end{tabular}

The largest cysteine framework (C8.0), was the most abundant framework for all species except for Homalonychus theologus. One framework (C12.1) was only recovered in Psechridae and Ctenidae. A novel cysteine framework (C10.1) not reported by Diniz et al. [16], was not recovered in P. nigriventer, though it was recovered in five other species of Ctenidae (Table 4).

Table 4: Number of ICK peptides recovered for each cysteine framework per species.

\begin{tabular}{lllllllll}
\hline Family & species & 6.0 & 8.0 & 10.0 & 10.1 & 12.0 & 12.1 & 14.0 \\
\hline Homalonychidae & Homalonychus theologus & 1 & 4 & 2 & 0 & 9 & 0 & 0 \\
Salticidae & Habronattus signatus & 6 & 13 & 2 & 1 & 1 & 0 & 0 \\
Xenoctenidae & Odo patricius & 2 & 21 & 2 & 0 & 3 & 0 & 0 \\
Anyphaenidae & Hibana sp & 3 & 10 & 0 & 1 & 1 & 0 & 0 \\
Gnaphosidae & Sergiolus capulatus & 1 & 11 & 0 & 1 & 2 & 0 & 0 \\
Thomisidae & Thomisus spectabilis & 2 & 13 & 3 & 0 & 2 & 0 & 1 \\
Thomisidae & Misumenoides formosipes & 1 & 4 & 0 & 1 & 0 & 0 & 0 \\
Oxyopidae & Oxyopes sp & 0 & 15 & 9 & 0 & 2 & 0 & 0
\end{tabular}




\begin{tabular}{lllllllll}
\hline Family & species & 6.0 & 8.0 & 10.0 & 10.1 & 12.0 & 12.1 & 14.0 \\
\hline Oxyopidae & Peucetia longipalpis & 1 & 11 & 4 & 1 & 0 & 0 & 0 \\
Lycosidae & Hippasa holmerae & 1 & 11 & 5 & 1 & 3 & 0 & 0 \\
Lycosidae & Pardosa pseudoannulata & 0 & 7 & 1 & 1 & 0 & 0 & 0 \\
Lycosidae & Schizocosa rovneri & 0 & 10 & 0 & 0 & 1 & 0 & 0 \\
Lycosidae & Sosippus placidus & 5 & 26 & 1 & 1 & 3 & 0 & 2 \\
Pisauridae & Nilus albocinctus & 1 & 8 & 2 & 1 & 3 & 0 & 0 \\
Pisauridae & Sphedanus quadrimaculatus & 1 & 6 & 2 & 1 & 4 & 0 & 0 \\
Pisauridae & Pisaurina mira & 0 & 1 & 0 & 1 & 1 & 0 & 0 \\
Pisauridae & Dolomedes triton & 1 & 11 & 0 & 0 & 10 & 0 & 1 \\
Psechridae & Fecenia protensa & 1 & 17 & 4 & 0 & 2 & 1 & 1 \\
Psechridae & Psechrus singaporensis & 0 & 13 & 3 & 1 & 2 & 1 & 0 \\
Ctenidae & Ctenus corniger & 11 & 19 & 5 & 1 & 3 & 1 & 1 \\
Ctenidae & Anahita punctulata & 2 & 15 & 1 & 0 & 2 & 1 & 1 \\
Ctenidae & Ctenus captiosus & 4 & 10 & 2 & 1 & 3 & 1 & 2 \\
Ctenidae & Ctenus exlineae & 2 & 9 & 2 & 1 & 2 & 1 & 1 \\
Ctenidae & Ctenus hibernalis & 2 & 10 & 3 & 1 & 3 & 1 & 1 \\
Ctenidae & Isoctenus sp & 3 & 11 & 4 & 0 & 0 & 1 & 2 \\
Ctenidae & Leptoctenus byrrhus & 1 & 12 & 2 & 1 & 1 & 1 & 1 \\
Ctenidae & Phoneutria nigriventer & 5 & 12 & 3 & 0 & 1 & 1 & 2 \\
\hline
\end{tabular}

Disulfide connectivity predictions Disulfide connectivity predictions varied greatly between the different prediction approaches; predictions for peptides with fewer cysteines were more consistent between approaches (Figure 2). The three disulfide bridges homologous to all cysteine frameworks were cross referenced to peptides with the same cysteine framework in Arachnoserver and used to guide the subsequent multiple sequence alignment (Figure 3).

Phylogenetic tests for selection: The first four inner cysteine loops shared by all ICKs were aligned following the schema defined in Table 5 . This resulted in a multiple sequence alignment with a width of 80 amino acids for 626 peptides with no redundant coding sequences and no sequences that failed the Chi-squared sequence composition test.

Table 5: Multiple sequence alignment schema for ICKs using the four pairs of structurally homologous cysteine residues.

\begin{tabular}{lllll}
\hline class & loop 1 & loop 2 & loop 3 & loop 4 \\
\hline $\mathrm{C} 6.0$ & $C_{1}-C_{2}$ & $C_{2}-C_{3}$ & $C_{4}-C_{5}$ & $C_{5}-C_{6}$ \\
$\mathrm{C} 8.0$ & $C_{1}-C_{2}$ & $C_{2}-C_{3}$ & $C_{4}-C_{5}$ & $C_{5} X C_{6}-C_{7} X C_{8}$ \\
$\mathrm{C} 10.0$ & $C_{1}-C_{2}$ & $C_{2}-C_{3} X C_{4}$ & $C_{5}-C_{6}$ & $C_{6} X C_{7}-C_{8} X C_{9}$ \\
$\mathrm{C} 10.1$ & $C_{1}-C_{2}$ & $C_{2}-C_{3}$ & $C_{4}-C_{5}-C_{6}$ & $C_{6} X C_{7}-C_{8} X C_{9}$ \\
$\mathrm{C} 12.0$ & $C_{1}-C_{2}$ & $C_{2}-C_{3} X C_{4}$ & $C_{5}-C_{6}$ & $C_{6} X C_{7}-C_{8} X C_{9}$ \\
$\mathrm{C} 12.1$ & $C_{1}-C_{2}$ & $C_{2}-C_{3} X C_{4}$ & $C_{5} X C_{6}-C_{7}$ & $C_{7} X C_{8}-C_{9} X C_{10}$ \\
$\mathrm{C} 14.0$ & $C_{1}-C_{2}$ & $C_{2}-C_{3} X C_{4}$ & $C_{5}-C_{6}$ & $C_{6} X C_{7}-C_{8} X C_{9}$ \\
\hline
\end{tabular}

Based on the reconstructed phylogeny of included ctenids (Figure 3) all other cysteine frameworks appear to have originated from framework C8.0. Framework C6.0 appears to have evolved via a loss of a pair of cysteines $\left(C_{6}\right.$ and $\left.C_{7}\right)$ from the original C8.0 framework. The largest monophyletic grouping of C6.0 was entirely unique to ctenids. The framework C10.1 represents an entirely separate lineage from C10.0 and is monophyletic. The remaining frameworks follow a general trend in terms of their evolutionary history, though they are not all completely monophyletic as some peptides from spurious cysteine frameworks were placed within what would otherwise be clades. 


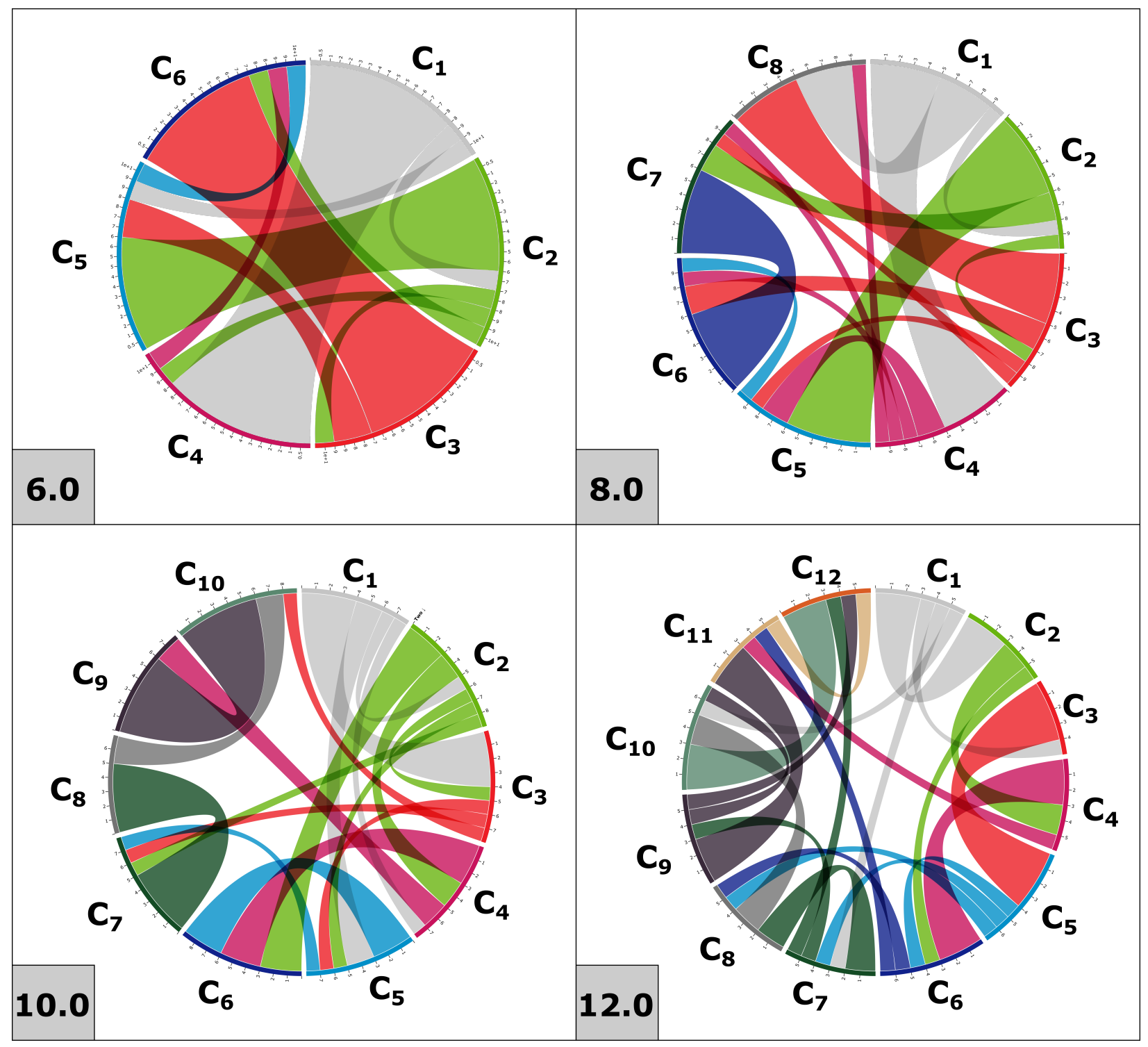

Figure 2: Pairwise disulfide connectivity predictions for cysteine motifs 6.0, 8.0, 10.0 and 12.0. Predictions come from a combination of four different prediction methods. 


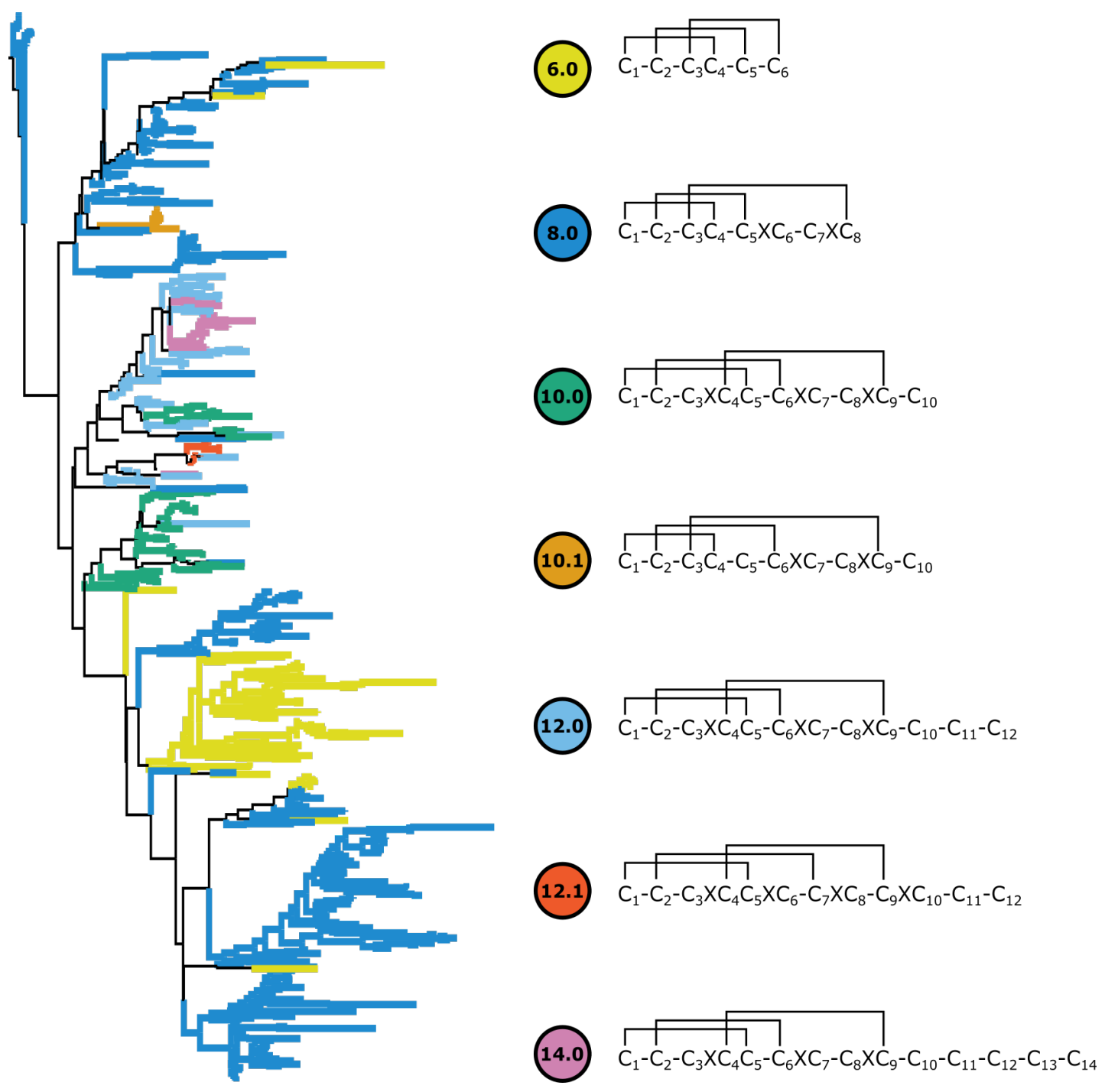

Figure 3: Reconstructed phylogeny of the 626 ICKs recovered from ctenids and lycosoid outgroups. Terminals are colored by their respective cysteine framework. Predicted disulfide connectivities representing all three homologous disulfide bridges shared among all ICK classes are shown to the right. 
BUSTED, with synonymous rate variation found evidence (LRT, p-value $\leq 0.05$ ) of gene-wide episodic diversifying selection across the entire gene phylogeny (Table 6). Therefore, there is evidence that at least one site on at least one branch has experienced diversifying selection. The site by site variation in test statistics is visualized in Figure 4, though BUSTED does not possess the statistical power to infer which specific sites or branches display evidence of episodic diversifying selection.

Table 6: A statistical summary of the models fit to the ICK alignment. "Unconstrained model" refers to the BUSTED alternative model for selection, and "Constrained model" refers to the BUSTED null model for selection.

\begin{tabular}{lllllll}
\hline Model & $\log$ (likelihood) & parameters & AICc & $\omega_{1}$ & $\omega_{2}$ & $\omega_{3}$ \\
\hline Unconstrained & -37648.7 & 1169 & 77691.4 & 0.06 & 0.09 & 3.35 \\
Constrained & -37733.9 & 1168 & 77859.6 & 0.03 & 0.03 & 1.00 \\
\hline
\end{tabular}

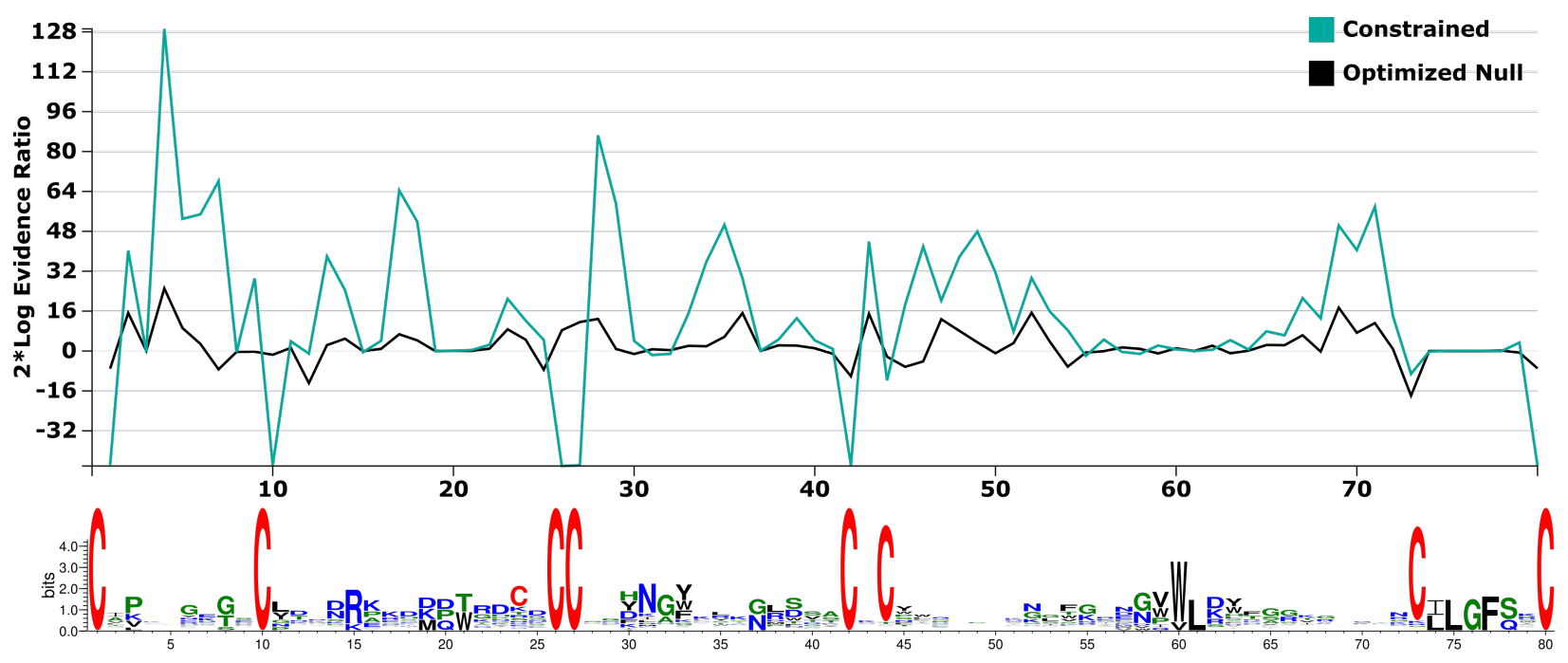

Site index

Figure 4: Model test statistics per site using $2 * \log$ evidence ratio for BUSTED constrained and optimized null. Sequence logos for the alignment are presented beneath.

FUBAR did not find evidence of pervasive positive/diversifying selection at any sites, but evidence of negative/purifying selection was detected at 57 sites with a posterior probability of 0.9 . The line of best-fit from a linear regression of $d_{S}$ as the independent variable and $d_{N}$ as the dependent variable for each of the 80 sites had a slope of $0.49\left(F_{1,78}=44.78, R^{2}=0.36, p=3.02 \times 10^{-9}\right)$. Only four sites had $d_{N}$ estimates that exceeded $d_{S}$, though the highest posterior probability of those sites was 0.54 (Figure 5).

MEME found evidence of positive/diversifying selection under a portion of gene phylogeny branches at 12 sites with p-value threshold of 0.05 , after correcting for multiple testing (Figure 6). Four were within the first loop between the first and second cysteine residues. None were within the second loop between the second and third cysteine residues. Two of those sites were directly upstream of the adjacent pair of cysteines (sites 26 and 27 of the alignment).

aBSREL found evidence of episodic diversifying selection on two out of 1,158 branches in the gene phylogeny. Significance was assessed using the Likelihood Ratio Test at a threshold of $\mathrm{p} \leq 0.05$, after correcting for multiple testing. One branch was a clade of four peptides with the C8.0 cysteine framework expressed by one ctenid (C. corniger), two oxyopids (Peucetia longipalpis and Oxyopes sp.) and one psechrid (Fecenia 


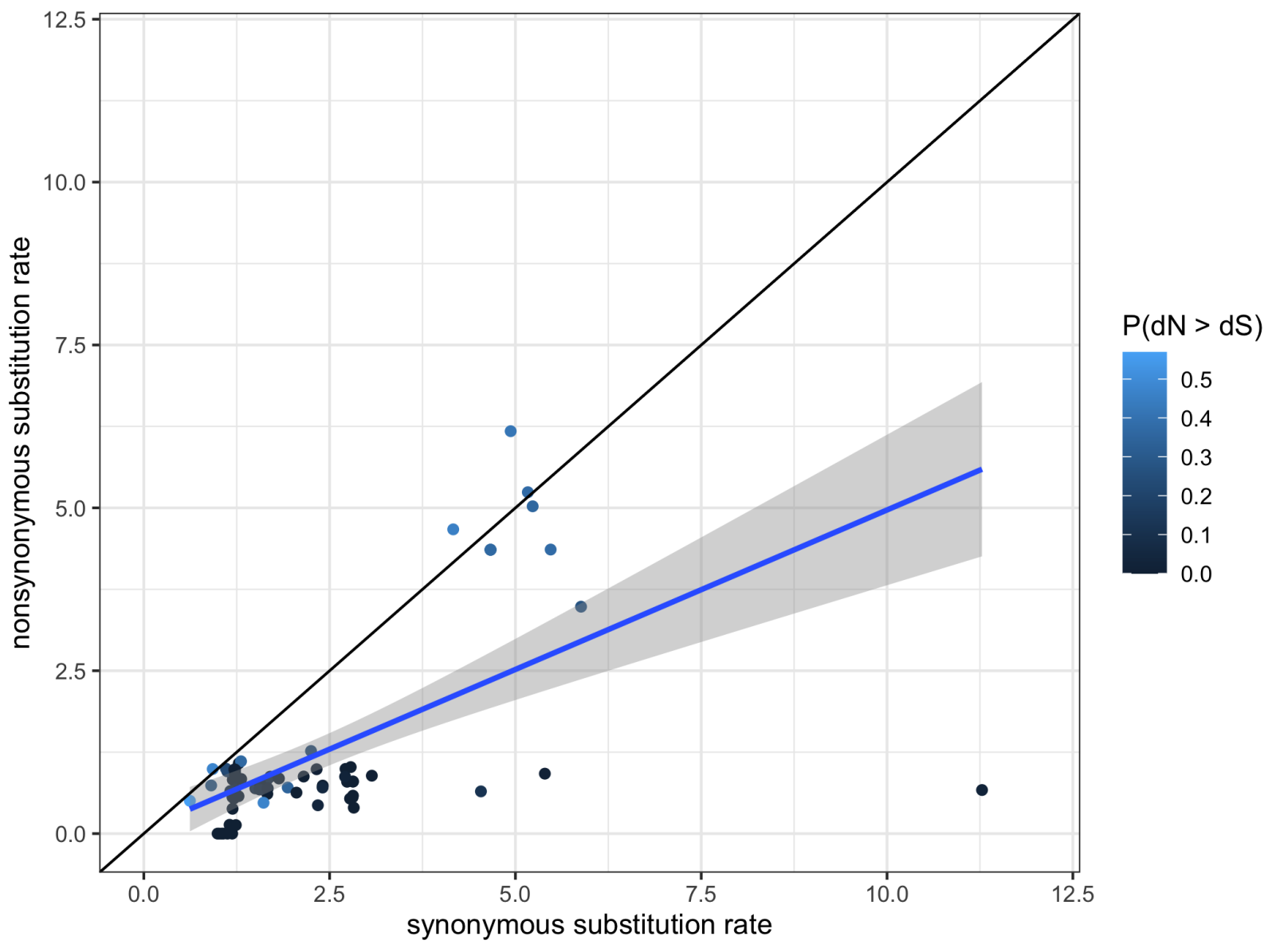

Figure 5: Scatter plot of synonymous substitution rate versus nonsynonymous substitution rate for each of the 80 sites of the ICK alignment. The black diagonal line indicates the null hypothesis of a lack of negative selection or positive selection where the two substitution rates are equal. Points are colored to indicate the posterior probability that a given site had evidence of pervasive positive selection. Line of best-fit is in blue, with the $95 \%$ confidence interval shaded in gray.

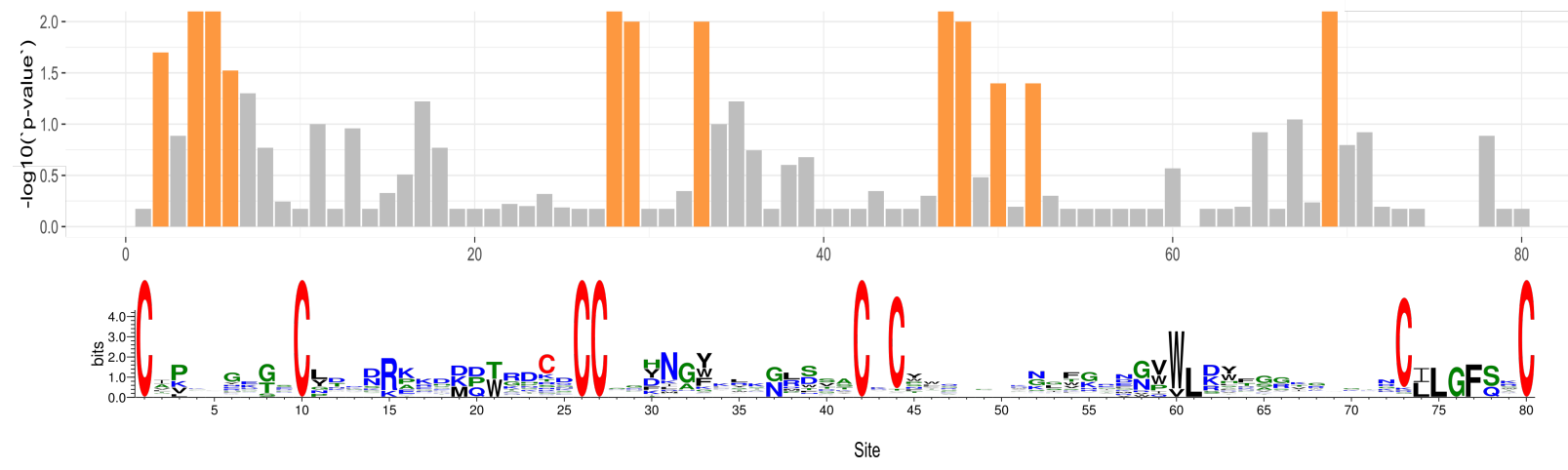

Figure 6: Bar plot of negative-log transformed p-values that a portion of branches for each site have evidence of episodic/diversifying selection. Sites with p-values $<0.05$ are highlighted in orange. Sequence logo for the alignment presented beneath. 
protensa). The other branch was a clade of 19 peptides with the C14.0 cysteine framework which comprises only only New World ctenids (i.e., excluding C. corniger).

BGM found 30 pairs of coevolving sites (posterior probability $\geq 0.95$ ), of which 10 had a posterior probability $\geq 0.99$. Of the 30 pairs of coevolving sites, 11 were at least three residues apart, whereas the furthest distance between two coevolving sites was 66 amino acid residues. Of particular interest was the fifth amino acid residue, which was found to be coevolving with three other residues, more than any of the others. Site 5, was found to be coevolving with sites 9,23 , and 35. Sites 5 and 9 are both found within the first loop between cysteines one and two, whereas site 23 is in the middle of the second loop, and site 35 is centrally located in the third loop (Table 7).

Table 7: Summary of amino acid coevolution analysis. Each of the 11 pairs of amino acid residues found to be coevolving by BGM that had a posterior probability greater than 0.95 , and were separated by at least 3 amino acids. Substitutions as well as substitution probabilities are listed for each amino acid pair.

\begin{tabular}{llllllll}
\hline$a a_{i}$ & $a a_{j}$ & $\mathrm{P}\left(a a_{i} \rightarrow a a_{j}\right)$ & $\mathrm{P}\left(a a_{j} \rightarrow a a_{i}\right)$ & $\mathrm{P}\left(a a_{i} \leftrightarrow a a_{j}\right)$ & $a a_{i}$ subs & $a a_{j}$ subs & Shared \\
\hline 2 & 9 & 0 & 0.99 & 0.99 & 155 & 161 & 54 \\
4 & 70 & 0.01 & 0.95 & 0.96 & 265 & 163 & 80 \\
5 & 9 & 0.98 & 0.017 & 1 & 193 & 161 & 67 \\
5 & 23 & 0.95 & 0 & 0.95 & 193 & 76 & 37 \\
5 & 35 & 0.57 & 0.42 & 0.99 & 193 & 117 & 55 \\
7 & 71 & 0.01 & 0.98 & 0.99 & 201 & 165 & 65 \\
13 & 28 & 0.22 & 0.77 & 0.99 & 154 & 187 & 66 \\
28 & 43 & 0.97 & 0.01 & 0.98 & 187 & 193 & 73 \\
38 & 41 & 0.29 & 0.68 & 0.97 & 17 & 23 & 6 \\
44 & 73 & 0.009 & 0.98 & 0.99 & 22 & 21 & 8 \\
\hline
\end{tabular}

\section{Discussion}

In this study, we identified and characterized the molecular evolution of 626 unique coding sequences for ICK peptides in wandering spiders and their free-hunting lycosoid relatives. The molecular functionality of these toxins is still unknown for the most part. Increased efforts in neurophysiology assays and molecular modeling will allow broader insights into the evolution of the molecular targets of these toxins. The best disulfide connectivity servers currently available were incredibly imprecise at predicting disulfide connections in spider ICKs ${ }^{48-50}$. This is especially true for ICK structural elaborations with more than four cysteine pairs, illustrating the need for a spider-specific approach to elucidating structural predictions. Unfortunately, a large bottleneck in making that a possibility is empirical investigations in determining the structure of ICK elaborations in spiders.

It appears that the original ICK toxin in spiders may not be what is typically referred to as the "core" ICK cysteine framework with three pairs of cysteines. Instead, the most abundant cysteine framework with four pairs appears to be the original ICK toxin, and the 6-cysteine framework evolved from the 8-cysteine framework via a loss of a pair of cysteines. Though this study only focused on lycosoid spiders, 8-cysteine toxins appear to be the most abundant throughout the spider tree of life, so a more comprehensive analysis of ICKs across all spiders may yield the same results.

It is postulated that antagonistic co-evolution through predator-prey interactions has shaped venom function via reciprocal selective pressures in an evolutionary "arms race"66-69. Though in rattlesnakes, the predator was observed to be the locally adapted antagonist by being "evolutionarily ahead" of their prey ${ }^{70}$. It is unclear if spiders are also evolutionarily ahead of their prey, though the fact that they have colonized nearly every terrestrial habitat on earth suggests this may be the case ${ }^{71}$, at least at broad taxonomic scales. At the 
protein level, selective pressures on venom have been observed through patterns of rapid evolution of amino acid sequences ${ }^{72}$. More specifically, according to the Rapid Accumulation of Variations in Exposed Residues (RAVER) model of venom evolution, structurally important residues receive strong negative selection while there is a rapid accumulation of variation in the molecular surface of the toxin under a coevolutionary "arms race" scenario ${ }^{73}$. The coevolution of venom resistance in prey and increasingly potent venom in the predator are theorized to exert reciprocal selection pressures.

Broadly speaking, there was evidence of gene-wide episodic diversifying selection in ICK toxins of lycosoid spiders. There was no evidence of pervasive positive selection in any of the codon sites of the ICK alignment. This is consistent with what has been reported in previous tests of pervasive positive selection in spider ICKs ${ }^{2}$. ICKs in spiders date back $\sim 300 \mathrm{MY}$, so it is not unusual that $\sim 70 \%$ of the amino acid sites demonstrated evidence of negative selection, because evolution "erases its traces" of early bouts of positive selection with persistent negative selection to preserve the potency of the toxin ${ }^{2,74}$. What is particularly striking, though, is that evidence of episodic positive selection was detected in a portion of branches for 12 amino acid sites. This is consistent with the two-speed model of venom evolution proposed by Sunagar \& Moran [2], in which positive selection pervades early in venom evolution (such as what is observed in the toxins of contemporary snakes and cone snails) followed by bouts of negative selection, then subsequent bouts of positive selection. These later episodic bouts of positive selection may be indicative of ecological specialization, such as dietary shifts and range expansions, resulting in a rapid diversification of venom arsenal.

Structurally, none of the residues between the first and second cysteine showed evidence of episodic diversification. This could indicate that those residues are necessary to maintain structural integrity and sustain venom potency. One of the two branches on the ICK phylogeny that had evidence of positive selection was a clade of 19 14-cysteine ICKs entirely unique to ctenids. It is possible that this ICK elaboration has played an important role in the range and diet expansion of ctenids. There was also strong evidence of amino acid co-evolution between one residue within the first loop and another amino acid four residues upstream in the same loop and two additional separate residues found midway through the second and third loop. This may indicate that these residues play an important role in the structural integrity or potency of the venom as they had a much higher than expected rate of co-occurrence.

\section{Conclusion}

In this study, we provided evolutionary insights into the ICK toxins of spiders. These insights may prove useful in the field of bioprospecting and peptide design, in which the ICK scaffold is useful for agricultural and pharmacological applications. What remains unresolved are the evolutionary mechanisms giving rise to molecular functions of these toxins, which will become a possibility as more structural and functional assays in spider ICKs are performed. None of the species included in our analysis have publicly available genome sequences, so our analyses relied on incomplete transcriptomic and proteomic data. However, we demonstrated that these toxins exist as multi-copy gene families across different species. What has yet to be determined are the specific mechanisms that have given rise to these large gene families. Sequencing the genomes of these spiders would provide valuable insights into the evolution of ICK toxins in spiders and finally allow investigations regarding the diversification and formation of cysteine framework elaborations of ICKs in spiders.

\section{Acknowledgements}

This research was made possible by the National Science Foundation Graduate Research Fellowship, the American Museum for Natural History Theodore Roosevelt travel grant, as well as East Carolina University's Department of Biology startup funds for the Brewer Lab. We also thank collaborators Drew Hataway, Brad Bennet, and Antonio Brescovit for providing tissue samples from Brazil. We thank Chris Cohen, Xinjun Wu, and Tim McDaniel for fieldwork assistance. 


\section{Data availability}

All short read data for this project can be found using BioProject accession number PRJNA587301, accessions for reads retrieved from SRA are in the supplemental data. All other data files are in the supplemental data files. Source code for this project is available on github (https://github.com/tijeco/killer_knots).

\section{References}

1. Walker, A. A. et al. Entomo-venomics: The evolution, biology and biochemistry of insect venoms. Toxicon 154, 15-27 (2018).

2. Sunagar, K. \& Moran, Y. The rise and fall of an evolutionary innovation: contrasting strategies of venom evolution in ancient and young animals. PLoS Genet 11, e1005596 (2015).

3. Casewell, N. R., Wüster, W., Vonk, F. J., Harrison, R. A. \& Fry, B. G. Complex cocktails: the evolutionary novelty of venoms. Trends in ecology \& evolution 28, 219-229 (2013).

4. Holford, M., Daly, M., King, G. F. \& Norton, R. S. Venoms to the rescue. Science 361, 842-844 (2018).

5. World Spider Catalog, W. World spider catalog, version 19.5. 2018.

6. Pineda, S. S. et al. Structural venomics reveals evolution of a complex venom by duplication and diversification of an ancient peptide-encoding gene. Proceedings of the National Academy of Sciences 117, 11399-11408 (2020).

7. Wong, E. S. \& Belov, K. Venom evolution through gene duplications. Gene 496, 1-7 (2012).

8. Schwager, E. E. et al. The house spider genome reveals an ancient whole-genome duplication during arachnid evolution. BMC biology 15, 1-27 (2017).

9. Escoubas, P. \& Rash, L. Tarantulas: eight-legged pharmacists and combinatorial chemists. Toxicon 43, $555-574$ (2004).

10. Arújo, D. A., Cordeiro, M. N., Diniz, C. R. \& Beirão, P. S. Effects of a toxic fraction, PhTx 2, from the spider Phoneutria nikriventer on the sodium current. Naunyn-Schmiedeberg's archives of pharmacology 347, 205-208 (1993).

11. Gomez, M. V., Kalapothakis, E., Guatimosim, C. \& Prado, M. A. Phoneutria nigriventer venom: a cocktail of toxins that affect ion channels. Cellular and molecular neurobiology 22, 579-588 (2002).

12. Nunes, K. P. et al. Tx2-6 toxin of the Phoneutria nigriventer spider potentiates rat erectile function. Toxicon 51, 1197-1206 (2008).

13. Richardson, M. et al. Comparison of the partial proteomes of the venoms of Brazilian spiders of the genus Phoneutria. Comparative Biochemistry and Physiology Part C: Toxicology 83 Pharmacology 142, 173-187 (2006).

14. Inns, R., Tuckwell, N., Bright, J. \& Marrs, T. Histochemical demonstration of calcium accumulation in muscle fibres after experimental organophosphate poisoning. Human $\mathcal{E}$ experimental toxicology $\mathbf{9}$, 245-250 (1990).

15. Mesilaakso, M. Chemical Weapons Convention chemicals analysis: sample collection, preparation and analytical methods (John Wiley \& Sons, 2005).

16. Diniz, M. R. et al. An overview of Phoneutria nigriventer spider venom using combined transcriptomic and proteomic approaches. PloS one 13 (2018).

17. Barrio, A. \& Brazil, O. V. Ein neues verfahren der Giftentnahme bei spinnen. Experientia 6, 112-113 (1950).

18. Munekiyo, S. M. \& Mackessy, S. P. Effects of temperature and storage conditions on the electrophoretic, toxic and enzymatic stability of venom components. Comparative Biochemistry and Physiology Part B: Biochemistry and Molecular Biology 119, 119-127 (1998).

19. Binford, G. J. \& Wells, M. A. The phylogenetic distribution of sphingomyelinase D activity in venoms of Haplogyne spiders. Comparative Biochemistry and Physiology Part B: Biochemistry and Molecular Biology 135, 25-33 (2003).

20. Clarke, T. H. et al. Multi-tissue transcriptomics of the black widow spider reveals expansions, co-options, and functional processes of the silk gland gene toolkit. BMC genomics 15, 365 (2014).

21. Chen, S., Zhou, Y., Chen, Y. \& Gu, J. fastp: an ultra-fast all-in-one FASTQ preprocessor. Bioinformatics 34, i884-i890 (2018). 
22. MacManes, M. D. The Oyster River Protocol: a multi-assembler and kmer approach for de novo transcriptome assembly. PeerJ 6, e5428 (2018).

23. Hart, T., Komori, H. K., LaMere, S., Podshivalova, K. \& Salomon, D. R. Finding the active genes in deep RNA-seq gene expression studies. BMC genomics 14, 778 (2013).

24. Longo, M. S., O'Neill, M. J. \& O'Neill, R. J. Abundant human DNA contamination identified in non-primate genome databases. PLoS One 6, e16410 (2011).

25. Lusk, R. W. Diverse and widespread contamination evident in the unmapped depths of high throughput sequencing data. PloS one 9, e110808 (2014).

26. Merchant, S., Wood, D. E. \& Salzberg, S. L. Unexpected cross-species contamination in genome sequencing projects. PeerJ 2, e675 (2014).

27. Bergmann, E. A., Chen, B.-J., Arora, K., Vacic, V. \& Zody, M. C. Conpair: concordance and contamination estimator for matched tumor-normal pairs. Bioinformatics 32, 3196-3198 (2016).

28. Edgar, R. C. UNCROSS: Filtering of high-frequency cross-talk in $16 \mathrm{~S}$ amplicon reads. bioRxiv, 088666 (2016).

29. Borner, J. \& Burmester, T. Parasite infection of public databases: a data mining approach to identify apicomplexan contaminations in animal genome and transcriptome assemblies. BMC genomics 18, 100 (2017).

30. Lafond-Lapalme, J., Duceppe, M.-O., Wang, S., Moffett, P. \& Mimee, B. A new method for decontamination of de novo transcriptomes using a hierarchical clustering algorithm. Bioinformatics 33, 1293-1300 (2017).

31. Ballenghien, M., Faivre, N. \& Galtier, N. Patterns of cross-contamination in a multispecies population genomic project: detection, quantification, impact, and solutions. BMC biology 15, 1-16 (2017).

32. Patro, R., Duggal, G., Love, M. I., Irizarry, R. A. \& Kingsford, C. Salmon provides fast and bias-aware quantification of transcript expression. Nature methods 14, 417 (2017).

33. Haas, B. J. et al. De novo transcript sequence reconstruction from RNA-seq using the Trinity platform for reference generation and analysis. Nature protocols 8, 1494 (2013).

34. Davidson, N. M., Hawkins, A. D. \& Oshlack, A. SuperTranscripts: a data driven reference for analysis and visualisation of transcriptomes. Genome biology 18, 148 (2017).

35. McIlwain, S. et al. Crux: rapid open source protein tandem mass spectrometry analysis. Journal of proteome research 13, 4488-4491 (2014).

36. Haas, B., Papanicolaou, A., et al. TransDecoder (find coding regions within transcripts). Github, nd https://github. com/TransDecoder/TransDecoder (accessed May 17, 2018) (2015).

37. Simão, F. A., Waterhouse, R. M., Ioannidis, P., Kriventseva, E. V. \& Zdobnov, E. M. BUSCO: assessing genome assembly and annotation completeness with single-copy orthologs. Bioinformatics 31, 3210-3212 (2015).

38. Katoh, K. \& Standley, D. M. MAFFT multiple sequence alignment software version 7: improvements in performance and usability. Molecular biology and evolution 30, 772-780 (2013).

39. Capella-Gutiérrez, S., Silla-Martínez, J. M. \& Gabaldón, T. trimAl: a tool for automated alignment trimming in large-scale phylogenetic analyses. Bioinformatics 25, 1972-1973 (2009).

40. Nguyen, L.-T., Schmidt, H. A., Von Haeseler, A. \& Minh, B. Q. IQ-TREE: a fast and effective stochastic algorithm for estimating maximum-likelihood phylogenies. Molecular biology and evolution 32, 268-274 (2015).

41. Mirarab, S. et al. ASTRAL: genome-scale coalescent-based species tree estimation. Bioinformatics 30, i541-i548 (2014).

42. Gelly, J.-C. et al. The KNOTTIN website and database: a new information system dedicated to the knottin scaffold. Nucleic acids research 32, D156-D159 (2004).

43. Altschul, S. F. et al. Gapped BLAST and PSI-BLAST: a new generation of protein database search programs. Nucleic acids research 25, 3389-3402 (1997).

44. Finn, R. D., Clements, J. \& Eddy, S. R. HMMER web server: interactive sequence similarity searching. Nucleic acids research 39, W29-W37 (2011).

45. Armenteros, J. J. A. et al. SignalP 5.0 improves signal peptide predictions using deep neural networks. Nature biotechnology 37, 420-423 (2019).

46. Miele, V., Penel, S. \& Duret, L. Ultra-fast sequence clustering from similarity networks with SiLiX. BMC bioinformatics 12, 116 (2011). 
47. Rubinstein, R. \& Fiser, A. Predicting disulfide bond connectivity in proteins by correlated mutations analysis. Bioinformatics 24, 498-504 (2008).

48. Ceroni, A., Passerini, A., Vullo, A. \& Frasconi, P. DISULFIND: a disulfide bonding state and cysteine connectivity prediction server. Nucleic acids research 34, W177-W181 (2006).

49. Yang, J., He, B.-J., Jang, R., Zhang, Y. \& Shen, H.-B. Accurate disulfide-bonding network predictions improve ab initio structure prediction of cysteine-rich proteins. Bioinformatics 31, 3773-3781 (2015).

50. Liu, Z.-L., Hu, J.-H., Jiang, F. \& Wu, Y.-D. CRiSP: accurate structure prediction of disulfide-rich peptides with cystine-specific sequence alignment and machine learning. Bioinformatics 36, 3385-3392 (2020).

51. Bostock, M., Ogievetsky, V. \& Heer, J. D $\mathrm{D}^{3}$ data-driven documents. IEEE transactions on visualization and computer graphics 17, 2301-2309 (2011).

52. Herzig, V. et al. ArachnoServer 2.0, an updated online resource for spider toxin sequences and structures. Nucleic acids research 39, D653-D657 (2010).

53. Shafee, T. M., Robinson, A. J., van der Weerden, N. \& Anderson, M. A. Structural homology guided alignment of cysteine rich proteins. Springerplus 5, 1-7 (2016).

54. Löytynoja, A. in Multiple sequence alignment methods 155-170 (Springer, 2014).

55. Pond, S. L. K. \& Muse, S. V. in Statistical methods in molecular evolution 125-181 (Springer, 2005$).$

56. Murrell, B. et al. Gene-wide identification of episodic selection. Molecular biology and evolution 32, 1365-1371 (2015).

57. Murrell, B. et al. FUBAR: a fast, unconstrained bayesian approximation for inferring selection. Molecular biology and evolution 30, 1196-1205 (2013).

58. Murrell, B. et al. Detecting individual sites subject to episodic diversifying selection. PLoS Genet $\mathbf{8}$, e1002764 (2012).

59. Smith, M. D. et al. Less is more: an adaptive branch-site random effects model for efficient detection of episodic diversifying selection. Molecular biology and evolution 32, 1342-1353 (2015).

60. Poon, A. F., Lewis, F. I., Frost, S. D. \& Kosakovsky Pond, S. L. Spidermonkey: rapid detection of co-evolving sites using Bayesian graphical models. Bioinformatics 24, 1949-1950 (2008).

61. Cheng, D.-Q. \& Piel, W. H. The origins of the Psechridae: Web-building lycosoid spiders. Molecular phylogenetics and evolution 125, 213-219 (2018).

62. Simó, M. \& Brescovit, A. D. Revision and cladistic analysis of the Neotropical spider genus Phoneutria Perty, 1833 (Araneae, Ctenidae), with notes on related Cteninae. BULLETIN-BRITISH ARACHNOLOGICAL SOCIETY 12, 67-82 (2001).

63. Davila, D. S. Higher-level relationships of the spider family Ctenidae (Araneae: Ctenoidea). Bulletin of the American Museum of natural History 2003, 1-86 (2003).

64. Brescovit, A. D. \& Simó, M. On the Brazilian Atlantic Forest species of the spider genus Ctenus Walckenaer, with the description of a neotype for C. dubius Walckenaer (Araneae, Ctenidae, Cteninae). Arachnology 14, 1-17 (2007).

65. Polotow, D. \& Brescovit, A. D. Revision of the neotropical spider genus Gephyroctenus (Araneae: Ctenidae: Calocteninae). Revista Brasileira de Zoologia 25, 705-715 (2008).

66. Van, V. \& Van Valen, L. A new evolutionary law. (1973).

67. Dawkins, R. \& Krebs, J. R. Arms races between and within species. Proceedings of the Royal Society of London. Series B. Biological Sciences 205, 489-511 (1979).

68. Endler, J. Defence against predators. Predator-prey relationships (1986).

69. Daltry, J. C., Wüster, W. \& Thorpe, R. S. Diet and snake venom evolution. Nature 379, 537-540 (1996).

70. Holding, M. L., Biardi, J. E. \& Gibbs, H. L. Coevolution of venom function and venom resistance in a rattlesnake predator and its squirrel prey. Proceedings of the Royal Society B: Biological Sciences 283, 20152841 (2016).

71. Turnbull, A. Ecology of the true spiders (Araneomorphae) (1973).

72. Juárez, P., Comas, I., González-Candelas, F. \& Calvete, J. J. Evolution of snake venom disintegrins by positive Darwinian selection. Molecular biology and evolution 25, 2391-2407 (2008).

73. Sunagar, K. et al. Three-fingered RAVERs: Rapid Accumulation of Variations in Exposed Residues of snake venom toxins. Toxins 5, 2172-2208 (2013).

74. Haller, B. C. \& Hendry, A. P. Solving the paradox of stasis: squashed stabilizing selection and the limits of detection. Evolution 68, 483-500 (2014). 